ARTICLES 


\title{
La presenza dell'Apocalisse nella cultura italiana ed europea tra medioevo ed eta'presente
}

\author{
Franco Cardini \\ Dipartimento Storia Medievali \\ Universitá di Firenze. Itallia
}

Resum. La presència de l'Apocalipsi a la culnura italiana $i$ earopea entre l'edat mitjana i el present

Resseguint el tema apocalíptic a través de diversos escrits, l'autor analitza la seva recepció a Itàlia i els seus usos religiosos i laics; tot atenent la relació amb diferents corrents religiosos, herètics i mil-lenarístics.

Paraules clau: Apocalipsi, De investigatione Antichristi, Ludus de Antichristo, heretgia, franciscans, Joaquim de Fiore, imperi, papat, apostòlics.

Abstract. Revelations' presence in Italian and European culture between Middle Age and present

Following apocalyptic theme across several texts, the author analyze its reception in Italy and its religious and lay uses; attending to its relation with various religious, heretical and millenaristic movements.

Key words: Revelations, De investigatione Antichtisti, Ludus de Antichristo, heresy, franciscans, Joachim of Fiore, empire, papacy, apostholic.

Forse a causa dell'approssimarsi della fine del esecondo millennioi dalla nascita del Cristo - o dalla data che tale è considerata tradizionalmente, nonostante sia ormai chiaro e noto che il computo secondo il quale essa fu stabilita era inesatto-, si vanno facendo sempre piu comuni appelli, paura, "profezie" connessi con l'attesa della fine del mondo e, con essi, i referimenti ad epoche del passato nelle quali una simile tensione, accompagnata da paure o speranze analoghe a quelle che oggi sembrano tornar a circolare, si era verificata. E si sente da più parti il bisogno di stabilire al riguardo analogie o di tracciar il confine delle differenze tipologiche e fenomenologiche tra istanze, fantasie, codici simbolici che paiono morfologicamente richiamarsi e tra i quali corre senza dubbio un sia pur sottile (e più volte rotto e riannodato) filo conduttore. Cerchiamo quindi di mettere un po' d'ordine in quest'intricata foresta di voci, d'immagini e di testi. 
Profetismo, pensiero escatologico, dimensione apocalittica, movimenti millenaristici: tutto questo attraversa la storia dell'Europa medievale, moderna e anche - sia pur attraverso una sorta di laicizzazione - contemporanea, intersecando in modi diversi la vicende sociali, la mistica, l'ecclesiologia, la politi$\mathrm{ca}$, la storia dei movimenti ereticali, l'astrologia, la magia. Tuttavia nella storia d'Europa c'è stato un momento - un lungo momento, estesosi per qualque decennio tra il XII e il XIII secolo- durante il quale l'Italia divenne terra d'elezione dei prophetae e dell'attesa dell'Apocalisse; è in questo momento decisivo che la peninsola ha visto sorgere i due personaggi che, in modi differenti e con ragioni diverse, sono stati senza dubbio i due maggiori protagonisti dell'apocalittica medievale: Gioacchino da Fiore e Francesco d'Assisi.

Il giochimismo italiano, forse un po' frettolosamente identificato da qualcuno con certe frange del francescanesimo spirituale, possiede al contrario una molteplicità di volti ed aspetti. La sua forza mistica riusci a coinvolgere uomini e movimenti molto differenti fra loro, fino a presentarsi in un certo senso come il presupposto dì una politica laica (si pensi agli ambienti da cui prese origine la politica di Federico I e poi di Federico II, con i suoi aspetti sacrali e millenaristici; ma, più tardi, anche a quelli che accompagnarono l'Imperatore Ludovico di Baviera, o all'attività ed al pensiero di Cola di Rienzo); misterioso e libresco, seppe anche farsi popolare, scendere in strada, animare ed entusiasmare le folle. Senza Gioacchino non si può comprendere Pietro da Morrone, quel papa Celestino $\mathrm{V}^{1}$ che fu, durante tutto il XIV secolo, il grande rimpianto, il sogno impossibile del mondo cristiano nell'epoca in cui la Curia pontificia era residente ad Avignone; senza Gioacchino non si comprendono nemmeno le invettive del Petrarca, né l'apostolato del Savonarola.

A meglio entrare in quest' ordine di problemi, si deve ricordare - dal momento che il mondo celestiniano è ben radicato nella realtà centroitalicache ci sono molte Italie e che, in particolare, esiste una cesura profonda tra un Settentrione latino e poi latino-germanico, strettamente legato all'Europa, e un Meridione prima greco, poi bizantino oppure musulmano (per quanto conquistato nell'xI secolo dai normanni), proteso da secoli verso Oriente, i Balcani e l'Asia Minore.

In questo mondo composito circolavano, forse a partire dall'Alto Medioevo, gli scritti chiamati «sibillini», opuscoli profetici d'origine ebraica e protocristiana il più noto dei quali resterà durante tutto il medioevo quello attribuito alla Sibilla di Tivoli, la Tiburtina, che risale alla metà del IV secolo e riflette la polemica fra gli imperatori d'Oriente e d'Occidente, ma anche e soprattutto quella tra cattolici e ariani. L'imperatore Costantino, che secondo la Tiburtina restaura il mondo cristiano, appare come il modello dell'imperatore dei Tempi Ultimi cui s'ispirerà tutto il medioevo per la sua letteratura apocalittica le manifestazioni della quale costituiscono in realtà altrettanti esempi di propaganda

1. Cfr. ora P. Gounalu, Il papa contadino. Celestino Ve il suo tempo, Firenze, 1996; AA. VV., Il papa eremita. Celestino Ve la perdonanza dell'Aquila, Verona, 1996. 
politica. Appare evidente che l'attesa dell'Apocalisse si colloca alla fine della storia, tuttavia pur sempre nella storia; e che ciò alimenta speranze e passioni caratterizzate da una netta qualità politica ${ }^{2}$.

Fu nel corso dell'xi secolo che, tra i cosiddetti «terrori dell'Anno Millen (in gran parte, come sappiamo, una leggenda storiografica) e l'inizio del movimento crociato, quell'attesa dell'Apocalisse si fece spasmodica un po' in tutt'Europa ${ }^{3}$. Essa interessò tuttavia relativamente poco I'Italia: verso la fine di quel secolo solo qualche cronaca dell'Italia meridionale, oltretutto di dubbia autenticità, menzionava avvenimenti meravigliosi, prodigiosi, a causa dei quali alcuni pellegrini si sarebbero mossi verso Gerusalemme. In realtà l'Italia non conobbe vere crociate di quelle poi definite epopolari» nello snodo tra XI e XII secolo con l'eccezione, forse, della spedizione milanese guidata dall'arcivescovo Anselmo nella quale può cogliersi l'ultimo bagliore del movimenci della pataria. Per gli italiani la prima crociata fu una questione limitata ai principi guerrieri che attraversarono la penisola nel 1096 dirigendosi verso i porti delle Puglie; $e$ anche un affare per i mercanti i marinai e i pirati delle città marittime come Genova, Pisa, in seguito Venezia. Vi furono saccheggi e furti di reliquie (eufemisticamente definiti translationes), ai quali sarebbe ingiusto negare il fondamento di un'autentica tensione religiosa: tuttavia - questo è il punto- essi non erano o non sembravano legati ad alcun rinnovamento escatologico, all'artesa di una nuova Gerusalemme che sarebbe discesa dai cieli. Si trattava piuttosto, in Italia, di una caccia alla sacralità della Gerusalemme Terrestre e dei Luoghi Santi tramite anche gli strumenti della corsa alle reliquie e della riproduzione della cappella del Santo Sepolcro e del Calvario all'interno delle chiese.

A questo punto un'apparente eccerione, che potrebbe anche essere interpretata come una contraddizione, segna la tradizione apocalittica italiana. Ordinariamente le esplosioni apocalittico-millenaristiche si accompagnano a qualche grande crisi collettiva, politica, sociale o economica: sono i sintomi di un'inquietudine, di un malessere, di una crisi d'identità, del desiderio di affidarsi a un salvatore o di definire un nemico metafisico che è necessario espellere o perseguitare, o entrambe le cose. Ma in Italia ad una prima analisi potrebbe dirsi che le cose non andarono in tal modo. Se accettiamo di considerare che le vere, nuove radici dell'attesa dell'Apocalisse sul suolo italiano nascono con Gioacchino da Fiore e Francesco d'Assisi, dobbiamo prendere atto che esse si sono impiantate in uno dei periodi più prosperi, sereni e soprattutto sicuri dell'intera storia dell' Italia medievale. Certamente difficoltà e guerre non mancavano: ma l'Italia di quel tempo appariva prospera, lanciata alla conquista dei mercati mediterranei (e secondo alcuni storici dell'economia si

2. Cfr. Norman CoHN, I fanasici dell'Aporalisse, Milano, 1976, p. 37-83; sulle profezie relative all'Imperatore dei Tempi Ultimi vedi F. KAMPERS, Die dewtsche Kaiseridee in Prophetie und Sage, München, 1986. Per un'analisi di tipo generale, che partendo dall'apocalittica ebraica attraversa la storia fino ai nostri giorni, cfr. B. MCGINN, L'Anticristo, Milano, 1966.

3. COHN, I fanatici, p. 66. 
raggiunse allora l'apogeo del suo sviluppo economico). L'Italia centrale e settentrionale era stata sufficientemente pacificata dopo il trattato di Costanza del 1183 tra Federico I e i Comuni, il papato era guidato dalla mano ferma e dalla lucida intelligenza di Innocenzo III, mentre il regno del Sud s'apprestava a passare dal governo normanno a quello degli Svevi.

$\mathrm{Ma}$ forse proprio all'interno di questa pace, di questa prosperità, si trova la chiave per comprendere veramente il senso dell'attesa dell'Apocalisse. La Chiesa uscita dalla riforma dell' XI secolo si era ben presto rivelata più interessata della precedente alle cose terrene: se la vecchia Chiesa feudale, sotto l'egemonia dei laici, si limitava a godere dei beni di questo mondo la nuova, nata dall'azione di Gregorio VII e Alessandro III, faceva nel contempo di più e peggio, pretendendo di dettar legge nelle cose terrene. Da decenni i catari seminavano anche in Italia i germi della rivolta contro questo stato di cose: si è dimostrato a sufficienza come il catarismo fosse non un movimento religiosopopolare cristiano, ma una nuova religione a carattere dualistico uscita dalla grande matrice manichea; nondimeno esso si appoggiava largamente sull'apostolato popolare e sulla contestazione dei vizi degli ecclesiastici per allontanare da essi il popolo cristiano Un'autentica dimensione apocalittica animava la propaganda e l'immaginario dei catari, i quali chiamano la Chiesa «Prostitutas e "Bestia" ${ }^{4}$ termini ed immagini ripresi dall' Apocalisse di Giovanni ma anche diffusi negli ambienti popolari, per quanto in quest'epoca la conoscenza della Scrittura fosse molto limitata. La mitologia catara, cosi come fu importata in Italia dalla peninsola balcanica tramite testi d'origine bogomila come l'Interrogatio Johannis, era molto lontana dal cristianesimo, benché utilizzasse simboli ed forme di linguaggio d'origine cristiana: i due poli tra i quali essa si moveva erano il Genesi e l'Apocalisse.

Non intendiamo sostenere con ciò che l'attesa dell'Apocalisse fosse, in Italia o altrove, risultato dell'eresia catara: fu semmai il catarismo a dover il suo successo popolare al fatto che era uso servirsi di un linguaggio apocalittico noto a tutti, quello del testo dell'evangelista Giovanni, tanto celebre, terribile e pittoresco quanto difficile a capirsi. Pertanto, dopo lo sterminio dei catari ${ }^{5}$, era necessaria una nova scintilla per propagare l'incendio apocalittico ricollegandolo a una forte passione popolare. Esso si accese col gioachimismo.

"Vi sono dei sacerdoti che non si dedicano al ministerio della Chiesa e dell'altare, ma piuttosto alle opere dell'avarizia, della vanità e degli spettacoli: così que le chiese, le quali dovrebbero essere case di orazione, trasformano in teatri e riempiono con mimici spettacoli di rappresentazioni sceniche. Nei quali spettacoli, presenti e spettatrici le donne, essi compiono talora perfino l'iniquo mistero dell'Anticristo, non come si crede immaginaria figura, ma in verità, com'è possibile credere per quanto sta in loro... Che cosa c'è quindi da stu-

4. Cfr. G. VolPE, Movimenti Religiosi e sette ereticali nella societa medievale Italiana, Firenze, 1961, p. 64

5. Per la crociata anticatara e il suo rapporto con l'idea e la pratica di crociata in senso più ampio, cfr. G. G. MERLO, Contro gli eretict, Bologna, 1996, p. 11-49 c passsim. 
pirsi se anche costoro, ora, simulando nelle loro rappresenrazioni l'Anricrisro o Erode, non mentiscono come si suole fare a scopo di divertimento, ma veramente li pongono sulla scena, come coloro la cui vita non differisce troppo dalla ignobile vita dell'Anticristo? Ed è anche accaduto talvolta presso costoro, come siamo venuti a sapere, che colui che nei loro divertimenti aveva figurato come morto da resuscitare per opera del profera Eliseo, compuita la simulazione, sia morto per davvero. Così, un altro, presentato all Anticristo per essere da questi resuscitato, nella settimana successiva siamo venuti a sapere morì veramente e fu sepolto. E chi può sapere se anche tutte le altre simulazioni, e cioè l'effigie dell'Anticristo, i fantasmi dei demoni, la pazzia di Erode, essi non presentino veracemente?

Presentano altresì immaginosamente la culla del Bambino Gesù, il suo vagito infantile, l'aspetto matronale della Vergine Maria, la stella come un astro lucente, la strage degli Innocenti, il materno pianto di Rachele. Ma la divinità ed il volto severo della Chiesa aborriscono gli spettacoli teatrali, non badano a vanita o a dissennate follie nelle quali gli uomini si rammolliscono come donne, i chierici si mutano in soldati, gli uomini si trasfigurano in larve di demoni... eppure in mezzo a costoro non mancano uomini illustri per stirpe, famosi per la scienza delle lettere, colmi di ricchezze, splendidi per gli ornamenti del corpo e delle vestin ${ }^{6}$.

In questo passo del trattato De investigatione Antichristi, redatto nel biennio 1161-63, e forse databile più propriamente al 1161-62, il teologo e polemista Gerhoh canonico di Reichersberg ${ }^{7}$ sembra rasentare, per implacabile rigore, i toni del De spectaculis del grande Tertulliano. Si è al riguardo più volte notato - ed è fin troppo facile notare- come questo passo sembri sostanziarsi di uno sdegno non generico bensi puntuale, e riferirsi quindi non ad azioni sceniche in generale bensì a rappresentazioni specifiche, simili o addirittura coincidenti con due delle quali ci sono rimasti i testi: il Ludus scenicus de Nativitate Christi e il Ludus de Antichristo ${ }^{\beta}$. Partendo appunto dall'ipotesi che la polemica di Gerhoh si appuntasse su rappresentazioni e testi precisi, e non si riferisse a stereotipi fluidamente circolanti, Ezio Levi proponeva in un saggio del ' 34 di intendere le allusioni all'Antichristo come riferibili a un testo ben noto, quello del Ludus de Antichristo contenuto nel celebre codice dell'abbazia di Tegernsee ora alla Nationalbibliothek di Monaco?. Il passo di Gerhoh serviva al Levi come base per una proposta di datazione riguardante la composizione del Ludus individuate come date estreme di essa il 1155 -data

6. Gerhohi REICHERBERGENSIS, De Investigatione Antichristj, in Gerhohi REICHERBERGENSIS Praeposm, Opera battenus Juedizn, Ed. E. Scheibel-Berger, I, Linz, 1875, p. 25-37.

7. Per il quale Cfr. A. M. Lazzarino DFl. Grosso, Società e potere nella Germamiat del xa Secolo. Gerhob di Reichersberg, Firenze, 1974.

8. Entrambi $\mathrm{i}$ testi si possono vedere in traduzione italiana nel volume Teatro latino medievale, a cura di E. FRANCESCHINI. Milano, 1960, rispettivamente p. 205-29 e 265-95.

9. E. L.EVI, "La leggenda dell"'Anticristo" nel teatro medievaleo, Snudi medievali, n.5., XII, 1934, p. 52-63. 
dell'incoronazione imperiale di Federico I Barbarossa, identificato e peraltro ben identificabile nell' Imperator della prima parte del Ludus, che, deposta la corona nel Tempio di Gerusalemme, agisce nella seconda parte come Rex Teotonicorum - e il 1169 - anno dell'ascesa al trono di Germania di Enrico VI di Svevia, preso a termine ante quem sulla base di un passo del Ludus, nel quale ci si lamenta del fatto che, accanto all'imperatore, non segga ancora in trono un re di Germania ${ }^{10}$-, il Levi stringeva ulteriormente l'arco di date da lui proposte prendendo come probabile anno più alto il 1162 (presumibile data di composizione del trattato del canonico di Reichersberg) e individuando la fase storica corrispondente all'allusione circa un'ostilità fra l'imperatore e il re dei Franchi, anch'essa presente nel testo, nai fatti accaduti tra 1162 e $1166^{\mathrm{HI}}$. Gli argomenti del Levi venivano ripresi e corretti da Ezio Franceschini il quale, in un saggio del 1940, proponeva di sfruttare in modo più rigoroso il testo di Gerhoh collegandolo in maniera stretta al Ludus di Tegernsee e datando pertanto quest'ultimo al periodo compreso fra 1155 e 1161 , vale a dire tra l'incoronazione imperiale di Federico e la data più alta di composizione, o d'inizio di composizione, del De investigatione ${ }^{12}$.

$\mathrm{Ci}$ sembra perd che, cosi facendo, il Franceschini finisse con lo svalutare un po' troppo il contesto storico-culturale del Ludus e le impressionanti citazioni sia pur rielaborate (e non semplicemente allusioni), in esso contenute, relative alla politica e - più ancor- alla «teologia politican barbarossiane: citazioni che non si spiegano né si esauriscono affatto nel recupero del concetto di sovranità universale mutuato dal diritto giustinianeo restaurato in Occidente grazie al lavoro dei Maestri di Bologna ma che vanno ben al di là di esso, fino a investire in pieno una serie di fatti collegati con la trionfante e trionfale politica federiciana avviata nel novembre 1158 con la seconda dieta di Roncaglia e culminata nel dicembre 1165 con la solenne canonizzazione di Carlomagno in Aquisgrana nonché nell'agosto 1167 con la reiterazione della stessa cerimonia dell'incoronazione imperiale in Roma. Nell'arco di quel decennio - grande regista dei successi imperiali del quale (e grande corresponsabile dei presupposti dei problemi che su Federico si sarebbero presentati negli anni successivi) fu il cancelliere Rainaldo di Dassel- si verificarono fatri il cui significato va molto oltre quello di simbolo e sintomo: anziutto la translatio del 1164 da Milano a Colonia delle reliquie dei Magi, e quindi la canonizzazione stessa di Carlomagno. Atti entrambi ispirati a una "teologia politica» (l'espressione schmittiana s'impone perentoria e inevitabile) che ben a ragione potremmo tout court definire "teologia imperiale" e che ribadivano, sanzionandola, la sacralità dell' ufficio imperiale e il carattere di "Cristo del Signore" e di "Nuovo Cristo" di colui che cingeva la corona imperiale, mundi dominus - secondo l'espressione dell' Archipoeta- e lex animata in terris, figura insom-

10. Ibidem, p. 54 .

11. Per questo cfr. F. CardinI, Il Barbarossa, Milano, 1985, p. 228-70.

12. E. Franceschin, "Hl dramma latino del secolo XIr: it "Ludus de Antichristo" ", Rivista italiana del dramma 1,1940 , p. 328-52. 
ma e vicario insieme del Cristo kosmokrator. Lucida e perentoria già in sé e per sé, questa politica - che includeva un forte legame con l'idea di crociata e quindi di sovranità sulla Città di Gerusalemme, e si raccordava direttamente al tessuto escatologico del mito dell'Imperatore dei Tempi Ultimi- assumeva un valore ancora più esplicito in quanto avviata durante lo (e, in gran parte, anche a causa dello) scisma inaugurato nel 1159-60, e che del resto Federico aveva fatto il possibile per scongiurare ${ }^{13}$.

Riteniamo che l'eco di quella politica e di quelle concezioni intensamente maturate nel decennio 1158-67 e successivamente affievolitesi, per riacquistare vigore nel penultimo decennio del secolo si colga nettamente nel testo del Ludus quale ce lo tramanda il codice di Tegernsee; e che ciò consigli appunto a guardare a quel decennio come al periodo nel quale presumibilmente tale testo venne composto e nel quale esso era chiamato a servite, dal momento che a nostro modo di vedere, e pur con tutta la cautela del caso, non ci sembra affatto azzardato ipotizzare, nel Ludus, un intento propagandistico preciso, che peraltro non è detto fosse l'elemento determinante e prevalente (e tanto meno l'unico, né a livello dì realizzazione e di "resa spettacolare" né a livello d'intenzioni). Intendiamoci: questi elementi condurrebbero di per sé a una rivalutazione della tesi del Levi, a derrimento almeno parziale di quella del Franceschini dal momento che l'elaborazione dei temi più specificamente teologico-imperiali sembra appartenere al periodo $1164-65$ più che a quello precedente. Tuttavia e con riserva di tenere conto di altri fattori di datazione che provenissero, intrinsecamente o estrinsecamente, da più approfonditi studi del codice di Tegernsee quel che non ci convince, più che nelle rispettive tesi, nel metodo comune al Levi e al Franceschini, è la ricerca tanto puntigliosa quanto in ultima analisi povera di elementi davvero probanti delle possibilità di trovare, nel testo del Ludus e nel suo confronto con gli avvenimenti storici del XII secolo, un evento o una serie di eventi al quale poter ancorare la redazione di esso. Colpisce ad esempio come tanto il Levi cuanto il Franceschini, evidentemente convinti entrambi del rapporto fra il Ludus di Tegernsee e la pagina di Gerhoh, abbiano legato senza dubbi apparenti il problema eminentemente

13. Per i testi relativi alla tensione tra impero $\mathrm{c}$ papato al tempo di Federico $I$, cfr. R. W.A. Y. CarlyLE, Il pensiero politico medievale, II, tr. it., Bari, 1959, pp. 517-616; sul concetto barbarossiano di sacralità dell'impero, ancora utili le pagine di R. FOLZ, L'idée d'empire en Occident du Ve au Xvie siecle, Paris, 1953, p. 110-23 (ma fondamentalmente resta P. E. SChramm, Kaiser, Rom und Renovatio, Darmstadt, 1957). Per il rapporto fra escatologia, attesa messianica, ruolo dell' imperatore romano-germanico e crociata, cfr. anebe P. ALPHANDERY - A. DUPRONT, La Cristianità e lidea di crociata, tr. it., Bologna, 1974, p. 219-23; per l'imperatore dei Tempi Ultimi, cfr. anche il libro di N. COHN, I fanatici dell'Apocalisse, tr. it. n. ediz., Milano, 1976, p. 135-62, dove tuttavia si studia l'escatologia del mito federiciano collegato essenzialmente con Federico II. Sull'impero romano-germanico, inoltre, non si possono dimenticare due bellissimi saggi che non si occupano specificamente del problema in questa sede centrale, ma che risultano preziosi per una sua corretta contestualizzazione storica: E. SESTAN, *Sacro Romano Impero ed Europa*, in AA. VV., La comunità europea. Storia e problemi, Firenze, 1969, p. 11-26, e G. TABACCO, L'impero romano-germanico e la sua crisi (secc. X-XIV), in AA. VV., La storia. II - Il medioevo, Torino, 1986, p. 305-38. 
testuale della redazione del primo con quello della datazione della seconda; lasciando in ombra, o decisamente evitando, qualunque altra possibilità, prima fra tutte quella che Gerhoh non alluda affatto a un testo preciso identificabile per giunta con quello pervenutoci, bensi a rappresentazioni sceniche argomento del quale era l'Antichristo, e che possono ben essersi iterate più e più volte; e delle quali il nostro Ludus potrebbe essere solo un esempio, magari importanti e cospicuo, comunque il solo rimastoci; o, per dir ancora meglio, delle quali esso potrebbe essere soltanto una delle molte, numericamente indeterminate varianti. Stupisce, fra l'altro, che né il Levi né il Franceschini abbiano tenuto il dovuto conto dell'atmosfera non solo escatologica, ma propriamente crociata che promana dall'intero Ludus. e che rinvierebbe a un momento nel quale Federico mostrò di voler ricollegare una risorgente istanza teologicoimperiale a un progetto concreto di crociata. Tale momento va situato verso la metà degli Anni Ottanta del secolo, allorché cominciarono a giungere dalla Terrasanta notizie allarmanti sulla nuova offensiva islamica che stava minacciando il regno crociato e che avrebbe avuto suo esito nella battaglia di Hattin e nella caduta della Gerusalemme crociata nel 1187, causa dells terza crociata. Dal nostro canto, francamente, non abbiamo trovato nel testo del Ludus motivi che ci conducano a escludere che il Ludus di Tegernsee possa essersi proposto come spettacolo anche a livello propagandistico riassuntivo della politica imperiale di Federico - magari anche con allusione a fatti di alcuni anni prima: e gli Anni Ottanta furono un tempo di rinnovata tensione tra imperatore e pontefice, dopo la pace del 1177 - ma comportante la veicolazione di un preciso elemento da propagandare, la crociata attraverso la quale l'imperatore intendeva affermare di nuovo il suo ruolo di signore universale e il suo ruolo cristico-messianico.

Semmai, un altro dato emerge dal Ludus: e lo collega di nuovo con estrema energia al testo di Gerhoh ed al momento culturale del quale quest' ultimo è espressione. Esso si afferma con evidenza attraverso il confronto tra la pagina del De investigatione e un testo più o meno ad essa coevo e di essa ben piü noto: il Policraticus di Giovanni di Salisbury. In tale testo l'utilizzazione di un passo di Petronio introduce nella cultura medievale europea la metafora del mondo come teatro, la quale va ad affiancarsi a quelle - allora tradizionali- del mondo come libro e del mondo come corpo ${ }^{14}$. La testimonianza di Giovanni di Salisbury sembra straordinariamente importante in quanto comporta l'atrestazione di una nuova sensibilità nei confronti dello spettacolo teatrale: quella stessa che Gerhoh condannava e che, forse, al contrario, la spregiudicata - e per molti versi elaicas? - politica di Federico e del cancelliere Rainaldo (ammesso che il Ludus appartenga agli Anni Sessanta del secolo XII) utilizzava propagandisticamente, ai fini dell' organizzazione del consenso, della mobilitazione attorno alla bandiera imperiale, magari (nel caso che il Ludus

14. Cfr. M. Fumigaui Beonso Brocchiers, La bugie di Isotta. Immagini della mente medienale, Roma-Bari, 1987, p. 64. 
vada riferito agli Anni Ottanta) addirittura attorno al forte e ben disegnato nesso tra ufficio imperiale, funzione messianica $\mathrm{c}$ impresa crociata.

Ma veniamo al testo. Il fatto che se ne conosca un solo esemplare rende molto problematico l'ipotizzare che si trattasse di opera diffusa: ma non costituisce, di per sé, neppure una prova in contrario. Semmai —ed è questo che a noi in questa sede più interessa - il testo di Tegernsee non prova la diffusione di spettacoli sull'Anticristo, ma indicia come propaganda imperiale e teologia imperiale fossero profondamente penetrate nella cultura chiericale tedesca e fossero ritenute suscettibili di una diffusione al tempo stesso edificante e demagogica. La tematica del Ludus, tuttavia, non si è formata in un giorno né in pochi decenni, né può essere spiegata e compresa tutta e soltanto alla luce delle emergenze degli Anni Sessanta o di quelli Ottanta del xII secolo. Già in un fondamentale studio del 1882 il Meyer dimostrava come esso si rifacesse a un trattato De ortu et interitu Antichristi dedicato prima del 954 di Adsone di Luxeuil, più tardi abate di Montier-en-Der, a Gerberga sorella di Ottone $\mathrm{I}^{15}$. Il riferimento a un testo di età ottoniana, mantenuto evidentemente vivo nella tradizione della cultura chiericale del mondo svevo, rende ancora più evidente e meglio comprensibile il legame con i temi della translatio e della renovatio imperii, che la propaganda sveva attingeva - rinnovandoli e rafforzandolialla tradizione ereditata dalle esperienze imperiali sassone e francone. In tale tradizione l'imperatore romano-germanico è appunto mundi dominus secondo il dettato dell'Archipoeta; è il signore universale dinanzi al quale - come proclamava il cancelliere Rainaldo di Dassel nel concilio di Dóle- gli altri re dell'Europa cristiana sono soltanto reguli provinciarum, "reucci», amministratori delle varie provincie di quell'impero che resta concettualmente (e, più che l'impero d'origine ottoniana, è quello del diritto giustinianco che qui subentra a legittimare e sorreggere l'ardita immagine del cancelliere) unico e invisibile al pari della Santa Tunica di Treviri, la "Tunica inconsutilen. Tali potenti immagini tornano, vivificate da un soffio di autentica commozione escatologica, nella Curia Jesu Christi tenuta il 27 marzo 1188 (la quarta domenica di quaresima) nella cattedrale di Magonza, allorché l'imperatore non siede sul trono che gli spetta, che invece viene lasciato vuoto affinché lo si riconosca appartenente a colui al quale appartengono tutti i troni della terra, al Cristo rex regum. Il gesto di Federico, in tale circostanza, è profondamente coerente con quello dell'Imperator della prima parte del Ludus, che depone la sua corona nel Tempio di Gerusalemme e torna fra i suoi come semplice re dei tedeschi. La Curia Jesu Christi di Magonza aveva appunto come suo oggetto la recente caduta della Gerusalemme crociata e la necessità di organizzare una nuova spedizione: in essa Federico, il suo primogenito e i grandi del regno presero solennemente il segno della croce, pegno del voto di compiere il pellegrinaggio armato alla Città Santa.

15. Cfr. W. MEYFR, eDer Ludus de Antichristo und Bemerkungen über die lateinische Rytmen des XII Jahrhundertens, Sirzungberichte der k. Bayr. Akademie des Wissenschafien, Phil. Philol, Kl., 1882, pp, 1-192. 
Ma il testo di Tegernsee offre materia per una serie di indagini: e, tra le righe, mostra quanto sia veramente legittimo mettere a frutto sino in fondo l'indicazione di Giovanni di Salisbury, e vedere sul serio «el mondo come teatron: vale a dire indagare su come un Ludus recitato all'interno d'una chiesa -quelle pratiche che eccitavano lo sdegno di Gerhoh di Reichersberg- potesse costituire un momento nel quale si rifletteva collettivamente sul fato della Cristianità e dell' intera umana famiglia inteso come Storia Sacra e, al tempo stesso, sulla struttura del mondo. Il teatro, la chiesa e il mondo sono appunto i poli del Ludus.

Partiamo, per illustrare brevemente questo tipo di lettura, da un'ipotesi: quella cioè che il Ludus sia concepito per venir recitato in una chiesa, una di quelle caratteristiche chiese del mondo germanico dalle due absidi affrontate, una ad est - con l'altare - ed una ad ovest; e, nelle grandi chiese imperili, al centro di quest'ultima è il trono del sovrano. Nella stessa Cappella palatina d'Aquisgrana, a sttruttuira ottagonale — a somiglianza della basilica gerosolimitana dell' Ascensione e della moschea della Cupola della Roccia, che gli occidentali interpretavano come l'antico Tempio della Città Santail lato orientale è occupato dall'altare, quello occidentale dal trono imperiale. Si delineano così due ipotesi: prima, le varie azioni dei personaggi del Ludus si svolgono nelle due absidi e lungo le navate laterali, e il pubblico assiste assiepato al centro della Chiesa (una prospettiva e una soluzione che rovescerebbero quelle consuete del rapporto fra scena e pubblico, che vuole semmai la scena al centro e gli sguardi del pubblico convergenti verso di essa); spettatori di élite, mentre il popolo, assiepato fuori dell'edificio, non assiste al Ludus bensì soltanto agli ingressi dei personaggi, e ne trae la lezione derivante dal significato dei loro aspetti, dei loro ornamenti; dei loro abiti.

$\mathrm{Ma}$ a meglio comprendere il messaggio proposto dal complesso meccanismo di messinscena del Ludus, bisogna tener presente anche che esso ha come argomento la storia dei Tempi Ultimi e come scenario, prima del Tempio di Gerusalemme, tutto il mondo e l'intera storia dell'umanità. I simboli attivati dalle varie scene del Ludus chiamano pertanto in causa, in un gioco di complessa compresenza, sia le strutture geografiche sia quelle temporali del mondo e della sua storia. A questo punto bisogna idealmente sovrapporre, all'immagine di una chiesa a doppio coro come quelle cattedrali di Magonza o di Bamberga, il tracciato di un tipico mappamondo del secolo XII, quello delle carte cosiddette a $\mathrm{T}$ \%. In esse, il globo terraqueo viene raffigurato da un circolo - l'oceano- all'interno del quale due braccia d'acqua - un diametro orizzontale, che indica la linea ideale Nilo-Don, e un raggio verticale inferiore, che indica il Mediterraneo-, isolano tre porzioni di terra, simili ciascuna a grandi fette di focaccia ma la prima (la superiore, orientata verso est) semicircolare e grande quanto le altre due insieme, le quali sono separate dal braccio acqueo minore - il Mediterraneo- e figurano affiancate. La porzione grande, orientata verso est, rappresenta l'Asia; le due minori sono rispettivamente I'Europa, orientata a nord-ovest (quindi alla sinistra dello spettatore) e l'Africa, orientata a sud-ovest (quindi alla destra dello spettatore). 
Gli ingressi dei personaggi del Ludus rispettano la cronologia della storia universale, che è la storia della sovranità del paganesimo, quindi del suo confronto con l'ebraismo, infine della conversione al Cristo; $i$ luoghi della chiesa ambiente nel quale si recita il Ludus nei quali ciascun personaggio si accomoda sono quelli corrispondenti alla diposizione che a ciascuno di essi spetta sul piano dell'ideale mappamondo costituito dalla chiesa stessa con le sue due absidi ripettivamente orientate a est e a ovest. Recíta difatti cosi la disposizione della scena e dei personaggi:

Templum Domini et septem sedes regales primum collocentur in hunc modum: ad orientem Templum Domini; huic collocantur sedes regis Hierosolimorum et sedes Sinagoge; ad occidentem sedes imperatoris Romani; huic collocantur sedes regis Theotonicorum et sedes regis Francorum; ad austrum sedes regis Gregorum; ad meridiem sedes regis Babilonie et gentilitatis ${ }^{16}$.

La sistemazione geomimetica dell'insieme non potrebbe essere più chiara $\mathrm{e}$ precisa. Che ad oriente vi sia Gerusalemme, quindi il re di tale città (si allude evidentemente al regno crociato fondato nel $1100 \mathrm{da}$ Baldovino di Boulogne) e gli ebrei, va de sé; e così che ad occidente vi siano l'imperatore - quindi Roma - ed i re di Francia e di Germania (ma il trono tedesco resta vuoto per turta la prima parte dell'azione scenica, finché non è l'imperatore, deposta nel Tempio la corona, e prendervi posto in quanto dopo il gesto compiuto a Gerusalemme non ha più diritto al trono imperiale sul quale è idealmente insediato, come nelle immagini di etimasia, il Cristo). Ma richiede forse una qualque spiegazione il seggio imperiale del sovrano dei greci (quindi dell'imperarore di Bisanzio) posto a nord (esso è effettivamente a nord, secondo la posizione relativa di Costantinopoli rispetto á Gerusalemme), e quelli della Gentilitas e del Rex Babyloniae, immaginati in Africa, quindi a sud (Babilonia si identificava correntemente con il Cairo). Si propone qui un'ambiguità comune alla cultura soprattutto laica del XII secolo, quella derivante dalla esplicita o implicita confusione tra il paganesimo grecoromano e l'Islam, in forza della quale i musulmani si rappresentavano politeisti e idolatri.

Fanno quindi ingresso per primi Gentilitas e Rex Babilonis, cantando le lodi della Deorum immortalitas, e si dispongono a sud. Tengono loro dietro Sinagoga e Indei, i quali cantano sì le lodi del Dio unico, ma nel contempo manifestano incredulità e disprezzo nei confronti del Cristo. Essi si dispongono a est.

Ecco a questo punto l'ingresso della Cristianità, con le sue figure allegoriche e i suoi massimi personaggi - riferiti tuttavia non a figure storiche concrete, ma alle loro funzioni-: è un ingresso scenografico, che bisogna immaginare svolgentesi a ovest o comunque diretto verso l'abside ovest della chiesa, che occuperà: mit deutschen Versen, hrsg. K. Langosch, Darmstadt, 1957, p. 182. 
Tunc Ecclesia in muliebri habitu procedit induta thoracem et coronata, assistente sibi Misericordia cum oleo ad dextram et Justicia cum libra et gladio ad sinistram utrisque muliebriter induris. Sequentur eciam eam Apostolicus a dextris cum clero et Imperator Romanus a sinistris cum milicia ${ }^{17}$.

Da notare il carattere allegorico delle figure di Ecclesia, Misericordia, Justicia, con i loro attributi c le loro posizioni: tutte cose che nella scultura c nella pittura non solo del XII, ma ancora fino al XV secolo $\mathrm{c}$ in molti casi anche oltre, vengono rigorosamente rispettati. Da notare ancora come, attorno alla Chiesa, papa e imperatore si dispongano rispettivamente a destra, dietro la Misericordia, e a sinistra, dietro la Giustizia, seguendo uno schema di separazione dei chierici dai laici e di rispetto d'una gerarchia destra-sinistra anch'essa di lunghissima durata (si pensi all'affresco di Andrea da Firenze per il Cappellone degli Spagnoli di Santa Maria Novella a Firenze). L'imperatore invia i suoi messaggeri rispettivamente al re dei francesi, dei greci e di Gerusalemme, che sono entrati nel frattempo: e l'iniziale superbia del re francese nei confronti dell'imperatore sembra alludere alle tensioni fra il Barbarossa e Luigi VII. Una rapida lezione militare sarà comunque sufficiente a far rientrare il re francese nei ranghi e a fargli accetrare - come avrebbe detto Rainaldo di Dassel-il suo ruolo di regulus provinciae. Il re dei greci e quello di Gerusalemme si sottomettono docilmente all' imperatore; sorge allora il Rex Babylonis, indignato per le vittorie e la gioria del nome cristiano. Egli attacca il Rex lerosolime, che chicde aiuto all'imperatore. Questi raccoglie le sue milizie, sconfigge il re di Babilonia, entra vittorioso in Gerusalemme e si spoglia nel Tempio delle insegne imperiali deponendole ai piedi del solo che può veramente dirsi Imperatore. $\hat{E}$, questa, la crociata intesa apocalitricamente, come aultima delle guerre» e condizione per I'aprirsi del Regno di Dio in terra. Ma proprio con l'umile gesto di remissione della corona, dopo un serrato dialogo tra Ecclesia, Gentilitas e Sinagoga, giunge e si insedia a Gerusalemme l'Anticristo, che - con puntuale parallelismo rispetto alla Chiesa - è circondato a destra dagli ipocriti (il falso clero), a sinistra degli eretici (i falsi cristiani, quindi i cattivi laici). Gli ipocriti depongono il re di Gerusalemme, che si rifugia presso il re dei tedeschi identificabile con colui che era stato, nella prima parte del Ludus, Y'imperatore. In quest'alleanza di falsi preti - gli ipocriti-con gli eretici, è possibile cogliere l'allegorizzazione dello scisma di Alessandro III visto dalla parte dell'imperatore? Se la datazione del testo agli Anni Sessanta fosse giusta, dovremmo ammeterlo. Ma nemmeno alla vigilia della partenza del Barbarossa per la terza crociata i rapporti con il papato erano troppo buoni. Si avvia quindi una elaborata fase di ambascerie dell'Anticristo ai re della terra, che tutti gli si sottomettono (la costruzione della scena è parallela a quella che aveva costituito la fase centrale della prima parte, con le sottomissioni all'imperatore all'imperatore). Solo il re dei tedeschi rifiuta di prestare omaggio all'Anticristo, che raduna 
contro di lui gli altri sovrani (un'altra allusione, peraltro venata di *eroico isterismon, alla effettiva situazione di Federico Barbarossa, che almeno nel decennio 1167-77 si trovò davvero isolato e circondato da un composito fronte di nemici che il papa aveva saputo drizzargli contro, ma che in parte la sua stessa condotta diplomatica gli aveva procurato? Si può vedere in tutto ciò un'almeno velata indicazione volta a lasciar capire che il vero Anticristo era il papa?). $S^{\prime}$ 'ingaggia quindi battaglia tra il re dei tedeschi e l'Anticristo: la vittoria arride al primo, ma il secondo - consigliato e assistito dagli ipocriti- si dà a compiere falsi miracoli. E il re tedesco, che a differenza dei suoi colleghi non si era lasciato piegare né dai doni né dalla forza delle spade, viene sedotto dalla falsa santità. Affiora in queste armi usate dall'Anticristo - la sia pur ingannevole forza taumaturgica, la potenza guerriera e la rícchezza che sa convincere per mezzo della corruzione - un interessante schema trifunzionale: l'Anticristo è veramente instauratore di un falso ordine che ha tuttavia apparenti caratteristiche di autenticità.

Restano immuni dalla sovranità dell'Anticristo i pagani e il re di Babilonia: com'è logico, dal momento che non riconoscendo il Cristo essi non possono accogliere chi pretende di venire nel Suo nome. Ed ecco che l'Anticristo affida proprio al re dei tedeschi, l'ultimo e il più puro - e quindi fedele- dei suoi seguaci, il compito di piegare i pagani in una nuova crociata. Invece alla Sinagoga l'Anticristo si rivolgerà direttamente: ed esse riconoscerà in lui l'Emanuele, il Messia. Sorgono però a questo punto i due profeti indicati dall'Apocalisse, Enoch ed Elia: sara la loro forte testimonianza e il loro martirio a far ricredere la Sinagoga. La Chiesa avrà parole di amoroso elogio per la resipiscenza della Sinagoga, che equivale a una conversione: a questo punto i tempi sono maturi per una rapida conclusione. Statim fit sonus super caput Antichristi, ed egli è repentinamente sbaragliato. Sulla trionfanti lode a Dio innalzata dalla Chiesa si chiude il testo del Ludus. Racconto dei Tempi Ultimi. o metafora del resto trasparente di vicendde politiche e religiose? Nel secondo caso, uno splendido esempio di propaganda politica attraverso il teatro. Una chiave parallela alla teologia imperiale per l'affermarsi del mito dell'Anticristo, è, come abbiamo detto, quella che si espande insieme al gioachimismo.

Col gioachimismo, certamente, ma senza dubbio non grazie all'abate Gioacchino. Questi rimane un esegeta duro e difficile, troppo oscuro e fissato sugli autori. Era un esegeta, non un profeta, anche se una tradizione, fatta propria in seguito dallo stesso Dante, I'ha celebrato come tale. Malgrado le sue origini e la sua fedeltà alla Calabria, una regione profondamente influenzata dalla cultura greca e dalla spiritualità bizantina, Gioacchino e il suo sistema trinitario (l'avvento delle erà successive del Padre, del Figlio e dello Spirito Santo) non si spiegano se non all'interno di un pensiero monastico rigidamente occidentale, il benedettino-cistercense, fondato sulla meditazione teologica e storica di Agostino per quanto aperto anche a influenze orientali, come dimostra il "cason della spiritualità camaldolese. La meditazione di Gioacchino, così come ci appare soprattutto nell' Expositio in Apocalypsim, è completamente 
opposta all'apocalittica immediata e volgare, e tende a concentrare in termini e medianti a metodi rigorosamente esegetici (che verranno semmai abbandonati o confusi nella posteriore pubblicistica gioachimita, che a livello testuale è in effetti pseudogioachimita) la propria ricerca sulla figura dell'Anticristo. Questi non e un unico personaggio, ma diversi: vi sono sette Anticristi, tanti quante le età nella storia e le persecuzioni sofferte dalla Chiesa, secondo l'immagine delle persecuzioni subite dal popolo d'Israele nell'Antico Testamento. Erode, Nerone, Costanzo, Cosroe, Enrico IV, il Saladino sono stati gli Anticristi del passato. Alla sua epoca Gioacchino attende il settimo e il peggiore degli Anticristi. Ma d'altra parte l'abate calabrese' si avverte contemporaneo al passaggio dalla quinta alla sesta età del Nuovo Testamento, che corrisponde all'attesa dell'Angelo del sesto sigillo dell'Apocalisse, colui che annuncerà i tempi nuovi e l'aurora della terza età del mondo, quella dello Spirito Santo. A lato dell'Angelo del steso sigillo vi sarà un Papa Angelico ${ }^{18}$ per annunciare questi tempi nuovi.

Il tormento esegetico dell'Apocalisse non è certo una novità nel mondo spirituale cristiano, e il lavoro di Gioacchino, malgrado tutta la sua originalità, non sarebbe sufficiente a spiegare la fama che lo circondo. Fu piuttosto la congiuntura religiosa e politica a innescare un processo la cui maturazione fu dovuta non tanto a Gioacchino quanto piuttosto agli scritti a lui attribuiti, simbolo ormai di una nuova speranza e iniziatore, tanto più temibile in quanto involontario, di una nuova ed ancor più pericolosa eresia.

L'attesa apocalittica appare anche legata ad una complessa esegesi matematica. Gli anni contano, e sono rigorosamente contati. Ad esempio, Gesù ha vissuto trentatré anni e gli apostoli erano docici come i mesi: ecco dunque che il 12 e il 33 acquistano un valore esegetico e cosmico; e nel XIII secolo si attende, con speranza e paura, l'anno 1233, l'anno del Cristo e degli apostoli. L'astrologia, studiata presso la corte sveva di Palermo e in quelle dei signori del nord Italia, fornisce alla profezia il suo supporto scientifico e si allea sovente con essa. Col Duecento si apre l'era dei pronostici, che accompagnerà per tre secoli la storia del continente, fino all'inizio del Cinquecento ed all'epoca di Lutero $^{19}$.

Non si conoscono bene, malgrado i numerosi studi dedicati all'argomento 20 i canali lungo i quali la ricchissima produzione di Gioacchino da Fiore s'arricchì di scritti pseudo-gioachimiti con un contenuto profetico sempre più

18. Cfr. S. Da Campagnola, L'angelo del Sesto Sigillon el'aAlter Christuss, Roma, 1971, p. $11-40$.

19. Nonostante la recentissima messe di studi federiciani, in quest'ordine di problemi resta da non dimenticare A. DE SteFANO, Federico II e le correnti spirituali del suo tempo. Parma, 1981. Sull Anticristo e Lutero, va richiamato Lutero, L'Anticristo. Replica ad Ambrogio Catarino (1521), a cura di L. RoNCHI DE MiCHel.IS, Torino, 1989, e A. Rotondo, "Ánticristo e Chiesa romana. Diffusione c metamorfosi d'un libello antiromano del Cinquecenton, in AA. VV., Forme e destinazione del messaggio religiosa. Aspetti della propaganda nel Cinquecento, a cura di $A$. Rotondo, Firenze, 1991. Ma cfr. anche infra, nota 52. 
esplicito. A questo proposito non può parlarsi di responsabilità esclusivamente francescane (i francescani non gradivano il processo di trasformazione dell'ordine in senso clericale e il suo allontanarsi dalla regola di vita propugnata da Francesco). Tuttavia senza alcun dubbio la grande popolarità raggiunta dai discepoli del Povero d'Assissi dopo il 1226, anno del suo transito, dà ragione del clima di tensione mistica complicato da un crescente impegno poitico (la lotta contro Federico II ed un ghibellinismo sempre più tinto d'eresia): questo è il clima che si respirava in tutt' Italia, e che era impensabile al di fuori della ricca vita sociale ed economica che si sviluppa nei comuni della Lombardia e della Toscana.

Nel 1233 nacque il movimento penitenziale dell'Alleluian, che si propagò in Italia centrale. Non sappiamo con certezza se fosse legato in qualche modo al gioachimismo: probabilmente no. Erano anni difficili, che videro la Chiesa ed i predicatori degli Ordini mendicanti lottare contro gli eretici cha a loro volta trovarono in qualche modo un oggettivo ancorché relativo e contradittorio appoggio politico in Federico II: egli d'altronde, se disponeva degli eretici come ottimi alleati nell'Italia dei comuni quando si trattava di combattere i guelfi ed il papato, perseguitava senza pietà ogni fermento eterodosso nelle terre sotto il suo controllo sicuro e diretto. Federico, protettore di quel frate Elia che gli Spirituali, ossia i francescani reclamanti la più stretta obbedienza al messaggio di Francesco, vedevano come il traditore del Povero d'Assisi (ma che Francesco pare aver amato e stimato), dal canto suo presentava una molteplicità di contraddizioni. Sembrava apprezzare, forse strumentalmente, il messagio di Francesco e l'idea di una Chiesa assolutamente povera non gli sarebbe spiaciuta, in quanto avrebbe facilitato i suoi progetti politici: nello stesso tempo però perseguizava gli Spirituali, che lo ripagavano definendolo Anticristo, e si proponeva come dominus mundi, Imperatore dei Tempi Ultimi. Tempi che sembravano ormai vicini soprattutto allorché, fra terzo e quarto decennio del secolo, i Tartari s'avventarono sull'Europa minacciando di conquistarla ${ }^{21}$.

Questo è il quadro storico in cui vennero elaborati gli apocrifi gioachimiti $\mathrm{e}$ in cui il ricordo legato all'esegeta Gioacchino da Fiore si mutò in venerazione nei confronti del profeta.

Come ha egregiamente dimostrato M. Reeves, verso il quarto decennio del XIrr secolo si defini il commentarío Super Fieremiam prophetam, che può essere considerato all'origine del successo degli apocrifi pseudo-gioachimiti ${ }^{22}$. Questo testo preparò il futuro sviluppo del gioachimismo, di fronte a una

20. Della sterminata bibliografia sull'argomento ci limitiamo a segnallare M. REEVES, The Influence of Prophecy in the Later Middle Ages, Oxford, 1969, e R. MANSELI, F. SimONI, E. PASZTOR, A. VOLPATO, G. TOGNETT, Ricerche sullinfluenza della profezia nel basso Medioevo, Roma 1973.

21. Cfr. D. Bigal.L, I Tartari e l'Apocalisse, Firenze, 1971, un saggio fondamentale.

22. Cfr. F. SimONI, $l l$ *Super Hieremiam e il gioachimismo framncescano*, in R. MANSELI, F. SimONI, E. PASzTOR, A. VOLPATO, G. TOGNETTI, Ricenche sull influenza, cit., p. 12-46; S. DA Campagnola, Dai Viri spirituales di Gioachino da Fiore ai Fratres spirituales di Francesco d'Assisi, Picenum Seraphicum XI, 1974, p. 24-52, 
gerarchia ecclesiastica venerata e oggetto di rimprovero a un tempo, minacciata dalla prospettiva di vedersi sostituita da una nuova Chiesa più pura e convinta che la purificazione le spetti con esclusivo diritto. In questo periodo un altro testo con influenze gioachimite, il Super Isaiam prophetam, condannava a sua volta l'imperatore Federico confermandone la tesi di un suo legame con I'Anticristo ${ }^{23}$. Ma l'influsso degli ambienti e degli scritti gioachimiti doveva esercitarsi allora ormai già da un certo tempo sul mondo francescano nell'Italia meridionale ed essere ben consolidato nel 1243-44, quando il cronista francescano Salimbene ci parla di un deposito di volumi concesso dai monaci florensi (l'ordine fondato da Gioacchino) ai francescani di Pisa ${ }^{24}$.

La congiunzione definitiva ed esplicita tra el francescanesimo-spirituale e il gioachimismo ebbe luogo nel 1254 , quando un giovane francescano che era stato, qualche anno prima, a fianco del famoso ministro generale spirituale Giovanni da Parma e che portava il nome di Gerardo da Borgo San Donnino pubblicò un Introductorium in Evangelium aeternum che riassumeva e comentava tre grandi opere dell'abate Gioacchinio: la Concordia Novi et Veteri Testamenti, l'Expositio in Apocalypsim e il Psalterium decem cordarum. Una commissione riunita prontamente ad Agnani, di cui faceva parte un cardinale d'origine domenicana, Ugo di Sant-Cher, procedette l'anno dopo alla condanna delle tesi esposte dal francescano ed anche, benché con minor durezza, delle tesi propriamente gioachimite: questa commissione fu senza dubbio impressionata dalle rimbombanti prese di posizione di un insegnante laico dell'Università di Parigi, Guillaume de Saint-Amour, che aveva violentamente attaccato Gerardo.

L'Introductorium ci è pervenuto attraverso una tradizione assai incerta e piena di lacune ${ }^{25}$ : due fonti, prodotte da schieramenti opposti, ce ne parlano ampiamente ma tendenziosamente, la Cronaca di Salimbene, scritta attorno al 1285 , e l'Historia septem tribulationem Ordinis Minorum, redatta tra il 1321 e il 1325 da Angelo Clareno, cioè da quel Pietro da Fossombrone che, dopo esser stato a lungo uno dei personaggi più in vista dell'ala francescana spirituale, passò alla congregazione degli eremiti Celestini fondata da Celestino V, contribuendo a creare un ponte tra Gioacchino, Francesco e Celestino. Questo collegamento, per quanto tendenzioso sarebbe stato per un lungo periodo l'asse portante del cristianesimo non eterodosso, ma nemmeno conformista, del XIV secolo.

Gerardo da Borgo San Donnino aveva annunciato che il 1260 sarebbe stato l'anno della rivelazione dei misteri divini ${ }^{26}$. E in effetti in quell' anno si diffu-

23. Cfr. H. DE Lubac, La posterità spirituale di Giachino da Fiore, I, Dagli spirimuali a Schelling, Milano, 1991, p. 98.

24. Da Campagnola, Dai Viri spiriruales, cit, p. 134.

25. B. TOPFER, Eine Handschrift des Evangelium aeternum des Gerardini von Borgo San Donnino",Zeitschrifi für Geschichtwissenschaft, VIII, 1960, p. 156-163.

26. R. MANSELLI, all 1260, anno gioachimitas, in AA. VV., II movimento dei disciplinati nel VII centenario del suo inizio, Perugia, 1962, p. 99-108. 
se a partire da Perugia, grazie all'iniziativa di Raniero Fasani, un movimento, detto dei Flagellanti o dei Disciplinati, caratterizzato da un atteggiamento nettamente penitenziale che Salimbene da Parma collega alle profezie di Gioacchino da Fiore ${ }^{27}$. I gruppi di flagellanti si spostavano da un luogo all'altro, da una città all'altra, sollevando ovunque entusiasmo e mettendo in serio imbarazzo i partigiani del ghibellinismo: anche se, com'è stato di recente ricordatto, «[...] i predicatori dell'Alleluia non erano tutti compattamente schierati politicamente e ideologicamente contro Federico IIs ${ }^{28}$. Il movimento si propagò a Bologna, Modena, Reggio, Tortona, Genova, nel Friuli ed anche fuori d'Italia. Nello stesso tempo, a Parma, Gherargo Segalelli fondava - ì sempre Salimbene che lo narra- un movimento detto degli “Apostolici» ${ }^{29}$. Il Segalelli era mosso dal desiderio di emulare Francesco d'Assisi, un'imitazione che secondo Salimbene giungeva ad aspetti grotteschi. Chiese di essere ammesso tra i francescani di Parma, ma costoro lo respinsero. Allora vendette tutto ciò che possedeva, lo distribuì ai poveri e, vestito unicamente di una miserabile tunica, iniziò a predicare, esortando a vivere seguendo letteralmente il Vangelo. Non sembra che abbia espresso un'autentica dottrina, e gli aApostolici” non si dotarono mai di una gerarchia. Ma formularono un giudizio assai duro contro il papato, che giudicavano corrotto, e contro la Chiesa, cui si rifiutavano d'obbedire e che rimproveravano perché non voleva riconoscere la perfezione del loro stato. $\mathrm{Si}$ presentavano come l'autentica Chiesa spirituale.

Gli Apostolici facevano propri i motivi che circolavano da due secoli all'interno dei movimenti religiosi popolari e pauperistici, e che non erano estranei al patrimonio valdese e francescano. E difficile dire se in tutto ciò potessero esservi echi propriamente gioachimiti ed elementi apocalittici, ma resta il fatto che la zona di Parma s'affermava come un area impregnata di fermenti di questo tipo: Borgo San Donnino, patria di Gerardo, non è lontana da Parma, patria del Segalelli.

Su almeno un punto gli Apostolici si rivelavano vicini alle idee gioachimite sull'Età dello Spirito Santo: il bisogno e il desiderio di un contatto diretto, immediato, non gerarchico, insomma libero con Dio. Era un'esperienza spirituale nuova vivamente in cerca di libertà interiore, che li differenziava nettamente dai loro contemporanei, $\mathrm{i}$ «Flagellanti», a carattere puramente penitenziale, la cui vocazione era il predicare la pace fra le città comunali lacerate dalle lotte tra fazion ${ }^{30}$.

Nel 1286 il papa Onorio IV ordinò agli Apostolici di entrare in uno degli Ordini mendicanti: era troppo tardi. Scattò allora il meccanismo della persecuzione, e i fascicoli degliinquisitori rivelano che - contrariamente a quanto

27. Cfr. R. MorgheN, „Raniero Fasani e il movimento dei Disciplinati del 1260», in Ibidem, p. $29-42$.

28. G. G. Merlo, Contro gli eretici, Bologna, 1996, p. 113.

29. Cfr. C. Violante, "Eresie nelle città e nel contado in Italia dall'xi al XIII secolos, in Idem, Studi sulla cristianità medievale, Milano, 1972, p. 370-371.

30. Ibidem, p. 375. 
è stato spesso sostenuto- il movimento degli Apostolici non era in origine pre-proletario e contadino. Al contrario, contava simpatizzanti nelle classi medie ed anche tra l'aristocrazia delle città. Si era esteso un po' dovunque nell'Italia centrale e settentrionale, sia nelle citta che nel contado, e se la Chiesa voleva sradicarlo non poteva riuscirci se non con l'uso della forza.

Verso il 1290 aderi agli Apostolici un oscuro personaggio nato presso Novara, Dolcino ${ }^{31}$. Ebbe quasi certamente rapporti diretti col Segalelli e quando costui fu arso, nel 1300, si trovava a Bologna: è possibile che si sia recato a Parma per assistere al rogo del maestro. In seguito vediamo Dolcino divenire il capo incontrastato degli Apostolici, se mai la parola capo ebbe per questa setta un senso: forse, nell'accezione weberiana, la setta non poteva darsi che un capo "carismatico" proprio in quanto era individualista e libertaria.

Guidati da Dolcino, gli Apostolici crearono un nucleo di resistenza armata e nello stesso tempo un embrione di quella che per loro era la società ideale in Valsesia, sulle Alpi: ormai si erano uniti ad essi contadini e montanari, anche se il movimento continuava a ricevere gli aiuti delle città comunali. Ma la resistenza in Valsesia non sembra aver avuto un carattere di rivolta sociale, nè un rapporto qualsiasi con la popolazione locale: $\mathrm{i}$ *dolciniani» restano dei déracinés, dei marginali. Per abbatterli definitivamente fu organizzata una crociata dai vescovi di Novara e di Vercelli: Dolcino fu catturato nel 1307 e quasi immediatamente bruciato sul rogo.

Dante esprime un giudizio molto duro su Dolcino, considerandolo eretico e fautore di scisma. Senza dubbio Dolcino disponeva di un'escatologia simplificata, adatta ad un movimento militante: presto sarebbe dovuto sorgere un Papa Santo (il Papa Angelico di Gioacchino, che alla fine del secolo molti avevano voluto riconoscere in Celestino V), poi un imperatore che avrebbe dovuto punire i falsi prelati della Curia romana (qui l'idea dell'Imperatore dei Tempi Ultimi si unisce ad una confusa volontà d'alleanza con i signori ghibellini dell'Italia settentrionale), ed infine doveva giungere l'ora dell'Anticristo e della lotta finale, nella quale solamente gli aderenti al movimento apostolico sarebbero stati salvati.

La dura repressione del movimento dolciniano non fu un caso isolato. Allo scoppio della tempesta catara la Chiesa aveva messo a pulito una serie di strumenti repressivi (pensiamo sopratturto all'Inquisizione ${ }^{32}$ ) che completavano la propaganda popolare condotta dai presicatori. Lo stesso movimento degli Spirituali francescani fu attacato con estremo rigore: due fra i maggiori esponenti di questa corrente, con Angelo Clareno, furono duramente perseguitati: il provenzale Pietro di Giovanni Olivi ed il piemontese Ubertino da Casale ${ }^{33}$. Un clima rovente, ormai quasi tragico (non è un gioco

31. Cfr. Fra Dolcino, a. c. di R. Orıot, Milano, 1984.

32. Su ciò, ancora una volta, la bibliografia italiana è troppo vasta: tuttavia non si possono non citare almeno le ricerche di R. ORIOLO, L'érsia a Bologna tra Xü e XIV secola, 2 voll., Roma, 1975.

33. R. Manselu, La *Lectura super Apocalypsim* di Pietro di Giovanni Olivi, Roma, 1955. 
di parole definirlo "apocalitticon) circonda le ultime gesta degli Spirituali francescani, ravvivate dalla straordinaria influenza che in Italia ebbe un testo come la Lectura in Apocalypsim, "l'ultimo sforzo, il più intenso, dell'escatologia medievale per presentarsi come una forza viva ed operante della Chiesa e nella storias ${ }^{34}$. Con questo testo, del resto, è necessario anche ricordare il già citato scritto del Clareno, l'Arbor vitae crucifixae Iesus dove con toni che ricordano più Gioacchino o i testi pseudo-gioachimiti che lo stesso Olivi, il parallelo tra Cristo e Francesco, concepito come un alter Christus, è condotto fino alle più estreme conseguenze ${ }^{35}$. Sappiamo che lo stesso Dante, a dispetto delle sue proteste d'imparzialità (che non fu mai il suo forte) condivise molte critiche degli «Spiritualin verso la Chiesa romana e nella sostanza aderì all'immagine di Francesco come alter Christus; attraverso il ghibellinismo delle posizioni politiche che assunse nell'ultima parte della sua tormentata esistenza, legato soprattutto all'esperienza dell'Imperatore Enrico VII, egli sembra avvicinarsi all'escatologia dell'Imperatore dei Tempi Ultimi ${ }^{36}$.

Pensiamo dunque che, tutto sommato, sia sbagliato credere che le idee ed i sentimenti collettivi di tipo apocalittico-escatologico s'inneschino a contatto con le realtà contingenti di tipo politico: in sé sono estranei ad esse, e nascono da una serie di sollecitazioni di natura mistica ed esegetica. Se questo genere di cose ha potuto esser vero, fino ad un certo punto, per Gioacchino da Fiore, resta il fatto che, dalla letteratura pseudo-gioachimita all'esperienza degli Spirituali francescani ed oltre, l'attesa dell'Apocalisse si nutrì di fermenti e di riferimenti politici immediati; il profetismo è fatto sovente di profezie-slogans, di profezie post eventum, insomma di profezie politiche. Di più: l'escatologiaa medievale (e dicendo questo non si toglie nulla al suo valore né al suo significato religioso e spirituale) è forse uno dei primi e più chiari esempi di mobilitazione delle folle in nome di una prospettiva di cambiamento collettivo. $\mathrm{La}$ realtà politica non è un supporto casuale, ma è veramente parte integrante dei sogni di rinnovamento legati all'escatologia. È vero d'altra parte che le contingenze rivestono una grandissima importanza nella diffusione dei vari movimenti apocalittici e millenaristici: essi restano legati ad una sensibilità cristiana popolare che rimane sempre nei confini dell'ortodossia e si manifesta in un senso soprattutto penitenziale (ad esempio il pellegrinaggio organizzato nel 1335 dal domenicano Venturino di Bergamo, o la Devozione dei Bianchi nel 1399), oppure legati ad una tenace istanza non conformistica che si radicalizza nel secolo del papato avignonese e dà luogo all'ultímo rigorísmo francescano o parafrancescano, il movimento dei fraticelli, o ancora ad esperienze più radicali come il movimento del Libero Spirito ${ }^{37}$.

\section{Ibidem, p. 236.}

35. Per una visione, oggi considerata con diverse perplessità, della scrittura e l'apparato immaginario-simbolico di Ubertino in connessione col Gioachimismo cfr. F. CALLAEY, Enede sur Uberrino de Casale, Louvain, 1911.

36. B. NARDI, Dante e la cultura medievale, Roma-Bari, 1983, part. p. 317-322.

37. AA. VV., L'attesa dell età nuova nella spiritualità della fine del Medioevo, Todi, 1962. 
Non intendiamo affatto proporre delle distinzioni rigide. Una devozione popolare ortodossa è spesso associata a temi di propaganda che possono essere contestatari nei confronti della Chiesa ed anche eretici: questa ibridazione può essere rintracciata all'interno di uno stesso ambiente ed anche di una singola persona: il XIV secolo, secolo di crisi socio-economica (dalla Peste Nera alla violenta depressione demografica, alle rivolte nelle citta e nelle campagne), è anche un secolo di crisi spirituale, per cui le condanne della mondanizzazione degli ecclesiastici, dell'eccessiva ricchezza e dell'immoralità della Curia pontificia appartengono ad un bagaglio culturale etico-polemico diffuso che deve essere esaminato caso per caso, al fine di identificarne i fattori specifici, e non attribuito affatto globalmente all'influenza di un testo o di un altro, di questo o quel gruppo di agitatori.

Ad esempio, sul piano politico vediamo i più estremi partigiani di una Chiesa povera e spirituale, $i$ efraticelliw, fornire il loro appoggio ad un imperatore il cui spessore morale è ben lungi dall'esemplarità: Ludovico di Baviera. Questi, nello stesso tempo, si trova depositario delle speranze in lui riposte da un teorico della politica come Marsilio da Padova, cui risalgono gli inizi di una concezione dell'impero ormai liberata dai presupposti sacrali tradizionalmente legati alla corona romano-germanica. D'altra parte una figura politica complessa come Cola di Rienzo, che ambisce a restaurare l'antica grandezza e libertà di Roma, nutre al tempo stesso una speciale devozione verso la memoria del Papa Angelico, Celestino (il Petrarca condivide in gran parte questa concezione) e inserisce elementi mistico-apocalittici nella sua propaganda politica $^{38}$.

Al tempo del papato d'Avignone, e ancor più durante gli anni seguenti - anni di scisma che videro la Cristianita divisa in due obbedienze pontificali- si può dire che la contestazione della mondalizzazione della Chiesa e l'attesa di un rinnovamento che sempre più coincideva con l'attesa della fine del mondo divenisse non più un'eccezione, bensì la regola. In una Cristianità percorsa dai predicatori, agitata da prodigi e visioni, diventava difficile distinguere con sicurezza tra idee ortodosse o eterodosse: meglio sarebbe forse parlare di atteggiamenti di tipo ereticale o no in quanto, al di là delle teorie, quel che soprattutto si manifestava era la volontà pratica di sottrarsi alla disciplina ecclesiale che definiva gli eretici come tali. Caterina o Bernardino da Siena non sono meno duri degli eretici nella loro critica verso la Chiesa visibile, né meno liberi di essi nel loro profetismo: tuttavia posseggono una ferma volontà di rimaner fedeli alla Chiesa ed al pontefice, volontà che li eleva al rango non solo di ortodossi, bensi di paladini dell'ortodossia.

Controversie fra i membri di una stessa fazione, legami ambigui ed ai limiti del rischio tra membri di fazioni opposte, sono dunque sempre possibili. L'attesa dell'Apocalisse, in Italia e altrove, riceve un contributo sostanziale dalle

38. E. DUPRE THESEIDER, "L'attesa escatologica durante il periodo avignonese, in ibidem", p. $98-105$. 
visioni di santa Brigida di Svezia e, a seguito di ciò, il mondo cristiano si popola di visionari: al punto che Caterina da Siena si erge duramente contro coloro che attendono senza posa l'Anticristo e in generale contro la fame di rivelazioni ${ }^{39}$. L'attesa continua dell'Anticristo, le voci secondo cui egli è già nato o è stato segnalato con ceertezza ${ }^{40}$, possono provocare dure reazioni ed essere ridicolizzate da uomini dotati da un buon senso robusto e forse abche un po' volgare associato ad una profonda religiosità: è questo il caso del romanziere e pio scrittore fiorentino Franco Sacchetti che malgrado qualche pressapochismo ci fornisce una vivace evocazione del clima del suo tempo: egli si lamenta in una delle sue composizioni poetiche dicendo che il mondo è pieno di falsi profeti, astrologhi, necromanti, e fa di ogni erba un fascio in quanto, nel concreto, tutte le attese e le visioni sembrano mescolarsi e confondersi ${ }^{41}$. Così, nell'atessa dell'Apocalisse e della nuova età, si mischiano i livelli, gli ambienti, le classi. Non sembra possibile, ad esempio, distinguere livelli cittadini o rurali, dominanti o subalterni, né leggere seriamente le attese apocalittiche come fenomeni di classe. Nel XIV secolo, e soprattutto dopo la grande crisi, le tensioni apocalittico-millenaristiche, i fermenti ereticali, le credenze magico-folkloriche sembrano sommarsi e fondersi in mille varianti disordinate. Si manifestano all interno delle classi più umili delle città ma anche nelle campagne e pure in taluni rappresentanti delle classi dirigenti; a Firenze, a Milano, nei grandi centri manifatturieri, ma anche nelle ragioni montane del Piemonte ${ }^{42} \mathrm{e}$ dell'Appennino umbro-marchigiano.

$\hat{E}$ obiettivaniente difficoltoso dare a queste credenze ed inquietudini precisi connotati sociopolitici e socioculturali, ma ciò non impedisce di rendersi conto che, nella loro complessità, esse erano giudicate pericolose per l'establishment, per il potere costituito. A Firenze il tumulto dei Ciompi (i lavoratori subalterni meno qualificati e più umili delle manifatture laniere) non s'era sviluppato nel 1378 all'insegna dell' Apocalisse: ma senza dubbio circolavano negli ambienti socialmente più bassi, i piccoli artigiani e i ceti che qualcuno ha voluto definire "preproletariv, simpatie per $\mathrm{i}$ afraticelli» le insegne che scelsero per le proprie assoziacioni corporative sembrano ricordare un simbolismo apocalittico: angeli armati di spada, braccia che escono dal cielo armate anch'esse di spada. Nel 1378 nel diario di un popolano fiorentino del quartiere d'oltrarno, troviamo una volgarizzazione del Vademecum in tribulatione di Giovanni da Roccatagliata $^{43}$; nello stesso anno, cosi instabile per la istituzioni fiorentine, il cancelliere Coluccio Salutati scrisse una lettera al suo omologo bolognese, Giuliano Zonarini, ossessionato da profezie e pronostici, dichiarando di non prestar fede alle previsioni astrologiche; anni dopo, sempre scrivendo allo Zonarini,

39. Rusconi, Lattesa della fine, Roma, 1979, p. 30.

40. Ibidem, p. $134 \mathrm{sgg}$.

41. Franco SACCHETT1, Il Libro delle rime, ed, a c. di A. CHIAR, BAR, 1936, p. 251.

42. Cfr. G. G. MERLo, Eretici e inquisitori nella sociera piemontese del Trecento. Torino, 1977.

43. Diario d'Anonimo fiorentino dall'anno 1358 al 1389, a c. di A. GHERARDI, Firenze, 1876 , p. 389-390. 
il Salutati tornerà sullo stesso tema per attaccare coloro che parlano della venuta dell'Anticristo: le due cose sembrano strettamente legate, dal momento che gli oroscopi religiosi e la scienza delle congiunzioni astrali lavorano per scoprire nei cieli i segni della venuta del terribile nemico ${ }^{44}$.

Del resto non era necessario che l'Anticristo fosse alle porte perché la sua presenza si manifestasse nella Cristianità. Sempre a Firenze Giovanni delle Celle, originario di Vallombrosa, consigliere spirituali di tutto un ambiente di pii membri dell'oligarchia, s'oppose alla penetrazione dei fraticelli, che giudicava partigiani dell Anticristo ${ }^{55}$. Ma nel 1374, anno in cui si scatenò un'altra grande epidemia, scrivendo a Guido del Palagio che gli aveva chiesto un parere attorno alle profezie relative alla fine del mondo, rispondeva che Dio non ne ha rivelato la data; tuttavia mostrava un devoto risperto nei confronti di Gioacchino da Fiore, cui sembra senza reticenze attribuire il dono della profezia, e considerava con attenzione l'opusculo Vaticinia de summis pontificibus, di cui ignora l'origine: questo in realtà viene dall'ambiente dei fraticelli ${ }^{46 !}$

L'attesa dell'Anticristo percorre ancora tutta la prima metà del XV secolo: fazioni contrapposte ne fanno un'arma all'interno della questione dell'obbedienza al papa. Orbene, essa diviene uno strumento politico: Bernardino da Siena, Manfredi da Vercelli, Andrea Biglia, Vicenzo Ferrer si scagliano addosso esegesi contrapposte e si rivolgono vincendevolmente l'accusa di essere precursori o discepoli dell'Anticristo ${ }^{47}$.

$\mathrm{Ma}$ è verso la metà del secolo, quando Costantinopoli cade nelle mani dei Turchi Ottomani - significativa coincidenza - che l'A pocalisse e l'Anticristo trovano un nuovo legame con la realtà storica immediata. Ma naturalmente, la connessione tra Anticristo e Islam non era cosa nuova.

Le voci che attribuivano a Maometto la natura dello scismatico, dell'eresiarca, del supporto del diavolo, erano correnti come sappiamo tanto in Bisanzio quanto in Occidente fin dall' VIII-IX secolo ${ }^{48}$. A Cordoba, nell'850, una cinquantina di cristiani fu fiustiziata per aver provocato le autorità musulmane: nella comunità cristiana nacque subito una polemica non soltanto sull'opportunità, bensì anche sulla legittimità della provocazione, innescata allorché un cristiano aveva accusato pubblicamente Maometto di essere uno dei «falsi Cristi» annunziati nella profezia evangelica ${ }^{49}$. Nella polemica, personaggi come Eulogio e Paolo Alvaro definirono maometto sangelo di Satana", "precursore dell'Anticristo», keresiarcan; e in particolare Paolo Eulogio, nel suo Indiculus luminosus, si appoggiava al commento di san Gerolamo a Daniele e di Gregorio Magno a Giobbe per identificare in Maometto sia l'undicesimo corno della

44. Rusconl, L'artesa della fine, Roma, 1979, p. 94-95.

45. Ibidem, p. 65.

46. Ibidem, p. 57.

47. Ibidem, p. 234-47.

48. Cfr. A. D'Ancona, La leggenda di Maometto in Occidente, n. ed. a cura di A. Borruso, Roma, 1994, part. p. 43 sgg.

49. Mt. 24, 16. 
quarta bestia ${ }^{50}$, sia i mostri Behemoth e Leviatan, tipi dell'Anticristo ${ }^{51}$. Maometto veniva quindi identificato con l'ultimo, il peggiore della linea di precursori e di prefigure dell'Anticristo, e Paolo Alvaro - insistendo sulla prossimità della fine dei tempi- dava segno di ritenere che Maometto, Islam e Anticristo coincidessero.

Secondo l'Expositio in Apocalipsim di Gioacchino da Fiore, II Saladino — che nel 1187 aveva strappato Saladino ai cristiani- era considerato la prefigura dell'Anticristo nella sesta fase dell'Età del Figlio ${ }^{52}$; ma un altro testo gioachimitico, il Liber Figurarum, proponeva una visione più complessa. Innocenzo III, scrivendo nel 1213 alla diocesi di Magonza nel quadro della preparazione di una nuova crociata, denunziava Maometto come il afiglio della perdizione» il regno del quale sarebbe durato 666 , dei quali tuttavia assicurava il pontefice- quasi seicento erano già trascorsi ${ }^{33}$. Nel famoso incontro tra Riccardo Cuor di Leone e Gioacchino da Fiore a Massina, nel 1191, il testimone diretto Ruggero di Hoveden riferisce che l'abate spiegò al re il contenuto dei capitoli 12 e 17 dell'Apocalisse in una maniera che rammenta l'esposizione della figura XII del Liber Figurarum: le sette teste del drago apocalittico sono i grandi persecutori della Chiesa, cinque dei quali (Erode, Nerone, Costanzo, Maometto e il "re» africano Melsemoth) sono già morti, mentre il sesto - il Saladino- è vivente, e il settimo deve ancora venire (ma secondo Gioacchino era già nato, e all'interno dell'impero romano) ${ }^{54}$. Maometto e Saladino si trovano tra i persecutori anche nello schema proposto nell' Expositio in Apocalypsim ${ }^{55}$. Riferimenti all'Anticristo in stretta relazione con l'Islam si hanno con frequenza in una serie di testi circolanti nel XIII secolo, dalla Profezia dei figli di Agap o Liber Clementis - un testo d'origine araba, ma diffuso nella sua versione latina e connesso con la crociata del $1217-1221^{56}$ - fino alla Profezia dei cedri del Libano composta nel 1239 in relazione con l'invasione mongola ma riscritta verso il 1290 sotto forma di profezia relativa alla caduta della Tripoli crociata $^{57}$. Ruggero Bacone, nel suo Opus maius composto verso il 1266-67, mettevca insieme profezie e calcoli astrologici per dedurne che la fine dell' Islam era prossima e che la conquista di Baghidad da parte dei mongoli ne era un segno. Nell' esegesi profetica duecentesca aveva naturalmente grande peso Gioacchino da Fiore e le opere sue

50. Dn, 7,8.

51. Pauli Alvar, Indicultes laminosus, 21 e 25-33, in P. L., CXXI, coll. 535, 539-1552.

52. Cfr. B. Toepfer, II regno futuro della liberta, Genova, 1992, p. 101.

53. Innocentii papae III, Registrum, 4725, cit. in P. Al.PHANDERY, eMahomet-Antichrist dans le Moyen Age latin", in AA. VV., Melanges Harnwig Derenbourg, Paris, 1909, pp. 263-65.

54. Cfr. B. McGinn, L'abate calabrese, Genova, 1990.

55. Ibidem, pp. 164-65.

56. Cfr. D. Bigalu, I Tartari e l'Apocalisse, Firenze, 1971, p. 187; cfr. TOEPFER, Il regno, cit., p. 233; per il testo della profezia, nel * Rois de Calabres annunziato dalla quale si potrebbe identificare Federico II, cfr. R. ROERICHT, Quinti belli sacri scriptores minores, Genève, 1879, p. 205 sgg.

57. MCGINN, L'Anticristo, cit. p. 208-9. 
o a lui attribuite ${ }^{58}$ : non il Gioacchino della dottrina trinitaria, bensì l'interprete dell'Apocalisse di Giovanni che aveva tracciato la storia dei capita diaboli, dei grandi persecutori, che secondo il cronista Ruggero di Hoveden erano, nella sequenza gioachimita, "Herodes, Nero, Constantius, Maumet, Melsemutus, Saladinus, Anticristus ${ }^{59}$.

Profetismo ed astrologia emersi nel Duecento, soprattutto grazie all'indagine di Ruggero Bacone appoggiata ad Albumasar, parvero tornar drammaticamente d'attualità a metà del Quattrocento. Commentanto Albumasar, Ruggero Bacone aveva computato come durata della Lex Mahometi 693 anni, un numero abbastanza prossimo al 666 apocalittico. E aveva preconizzato, dopo la «legge di Veneres ch'era quella dei licenziosi saraceni, l'avvento dell Lex Lunae, quella dell'Anticristo il simbolo del quale era appunto la luna in quanto pianeta dell'instabilità, della corruzione, della necromanzia, della menzogna ${ }^{60}$.

Fu nella luna ottomana che pertanto non si ebbe difficoltà - Ruggero Bacone aveva parlato della legge di Maometto come dell' ultima presentazione dei precursori, prima dell'avvento dell'Anticristo- a riconoscere i tratti del terribile nemico: ciò soprattutto dopo la caduta di Costantinopoli ${ }^{61}$. In Italia questa credenza diffusa, alimentata da una quantità di pronostici e da predicatori itineranti di provinienza spesso incerta ${ }^{62}$, unisce all'astrologia umanistica ed alla ricerca febbrile di segni celesti. Dopo la cometa del $1472^{63}$, Francesco da Meleto consulterà febbrilmente i saggi ebre ${ }^{64}$, mentre Lorenzo Bonincontri nel suo poema De rebus coelestis, scritto tra il 1472 e il 1475 , s'interrogherà con inquietudine attorno alla congiunzione tra Giove e Saturno ${ }^{65}$.

Ad Otranto nel 1480 una scorreria turca s'impadronì della città costiera pugliese, saccheggiandola e martirizzandone gli abitanti: il che sollevò nuovi timori ed anche nuovi entusiasmi, nuove proposte di crociata ${ }^{66}$. In quel momento responsi astrologici e movimenti spontanei d'entusiasmo religioso s'associavano o perlomeno convergevano nel mobilitare le coscienze. Nel dicembre

58. Per la tradizione gioachimita: R. MANSELU - F. SIMONI - E. PASZTOR - A. Volpato - G. TOGNETT, Ricerche sull'influenza della profezia nel Basso Mediocvo, Roma, 1973; H. DE LUBAC, La pasterita spirinuale di Gioacchinio da Fiore, voll. 2, Milano, 1979; AA. VV., Storia e messaggio in Gioacchino da Fiore, S. Giovanni in Fiore, 1980; H. GRUndmanN, Studi su Gioacchino da Fiore, Genova, 1989; B. MCGINN, Labate calabrese, cit.

59. Cfr. BigALU, I Tarrari, cit., p. 108; cfr. E. PISPISA, Gioatchino da Fiore e i cronisti medievali, Messina, 1988, ad indicem.

60. Rogerii BACONIS, Opus maius, 1, 261-62, cir, in BigAlL, I Tartari, cit., p. 184.

61. Cfr. La caduta di Costantinopoli, a cura di A. PERTUSI, voll. 2, Milano-Roma, 1976: A. PERTuSI, Fine di Bisanzio e fine del mondo, Roma, 1988.

62. Cfr. A. VOLPATO, «La predicazione penitenziale-apocalittica nell'attività dei due predicatori del 1473n, in MANSELI1, SIMONI, PASZTOR, VOLPATO, TOGNETT, Ricerche sull'influemza, p. 113-128.

63. Ibidem, p. 124.

64. Cfr. G. ToGNETT, “Note sul profetismo nel rinascimento e la letteratura relativan, in ibidem, p. 142.

65. Cfr. B. Soldatl, La poesia astrologica nel Quatrocento, Firenze, 1906, p. 160-162.

66. Cfr. C. VASOLI, I miti a gli astri, Napoli 1977, p. 46. 
dello stesso anno comparve il De futuris christianorum triumphis in saracenos, un testo meglio noto come Glosa super Apocalypsim. Esso fu edito l'8 dicembre 1480 dal carmelitano Battista Canale, ma ne era autore nella quale l'inquietante umanista domenicano Giovanni annio da Viterbo, il cui nome nel secolo era Giovanni Nanni. Insistendo sulla crociata, egli pubblicava i suoi pronostici sull'inevitabile caduta dell' Impero turco, e identificava a sua volta con certezza l'Anticristo con Maometto ${ }^{67}$. Bisogna ricordare che il titolo completo dell'opusculo è Glosa in Apocalypsim, ma che esso è più noto con quello di De futuris christianorum triumphis in Samceno ${ }^{68}$. Mentre si attendevano avvenimenti nuovi ed immediati, il cielo cristiano veniva in aiuto al cielo astrale: nel 1482 e 1484, per esempio, due grandi episodi miracolosi di segno mariano a Bibbona in Maremma ed a Prato vicino a Firenze, videro entusiasmarsi il popolo cristiano ed ispirarono ad esso una nuova forza rendendolo certo che qualcosa di straordinario stesse per giungere ${ }^{69}$.

Infine arrivò il grande anno, magnus annus, el 1484. Da lungo tempo era atteso come l'anno dei grandi cambiamenti secondo una lunga tradizione astro$\operatorname{logica}^{70}$ : a Firenze l'umanista Cristoforo Landino previde in quest'anno il ritorno di un personaggio enigmatico ed allegorico menzionato da Dante come cil Veltrow, i tratti escatologici del quale molto vicini all'Imperatore dei Tempi Ultimi $^{71}$. È sempre nel 1484 che ebbe luogo la misteriosa cavalcata del profeta Giovanni Mercurio da Correggio, portatore di visioni ispirate alla cultura ermetica da poco impiantata in Occidente, che nelle strade di Roma invitava a una generale penitenza ed annunciava una prossima renovatio ${ }^{72}$.

E nel clima apocalittico ravvivato da questi avvenimenti e solecitazioni che Girolamo Savonarola inizia la sua carriera di profeta ${ }^{73}$ durante la quale non mancherà la visione della spada di Dio uscente dalle nubi, secondo un simbolismo che a Firenze i Ciompi avevano fatto loro ed integrato nel loro sistema araldico piuttosto semplecistico. Si è molto parlato del carattere propriamente politico del profetismo del Savonarola e degli aspetti ideologici di alcune profezie diffuse per servire questa o quella causa: basti pensare al carattere francofilo della famosa profezia di Telesforo da Cosenza. Questa carica proferica - che il Savonarola condivide con altri celebri predicatori del suo tempo- è un elemento della sua ascesa verso lo stato di edittatoren spirituale (e non solamente spirituale) fino al 1498; ed è pure un elemento della sua caduta, tant'è vero che le folle odiano sempre coloro cha hanno troppo amato nel momen-

67. Cfr. G. M. Barbuto, Il principe e l'Ansicristo, Napoli, 1994, p. 40; importante per il rapporto fra tradizione gioachimita e pericolo turco, AA, VV., 11 profetismo gioachimita tra Quattrocento e Cinquecento, a cura di G. L. PODESTA, Genova, 1991, passim.

68. lbidem, p. 19.

69. Cfr. D. WeInSteIn, Savonarola e Firenze, tr. it., Bologna, 1970, p. 96.

70. Ibidem, p. 98

71. Ibidem, p. 99 .

72. Cfr. K. OHLy, Johannes Mercurius Corrigensis, in Beitrdge zur Inkunabelkunde, hrsg. C. Wehem, n. F., II, Leipzig, 1938, p. 133-147.

73. D. WEINSTEIN, Savonarola e Firenze, p. 89, 102. 
to in cui la fortuna sembra li abbandoni; e può esser vero, come diceva il Machiavelli, che questa è la fine dei profeti disarmati. Ma l'apocalisse del Savonarola non è solamente il Giudizio e i Tempi Ultimi: essa è anche - ciò che d'altronde fu sempre - I'inizio dell'era del ecielo nuovo e della nuova terras, il tempo dell'uscita dalla storia in cui la Gerusalemme celeste doveva scendere su questo mondo. Come hanno ben sottolineato più tardi i suoi fedeli descepoli, come Giovanni Nesi, era questa Gerusalemme che il domenicano ferrarese voleva instaurare a Firenze, per mezzo delle sue riforme costituzionali: il regno della giustizia, la Nuova Gerusalemme. Illusioni, certamente, del tutto tragiche. L'Apocalisse è la madre di tutte le utopie moderne, comprese le più abiette, le piü sanguinose.

Nel 1492, anno in cui morì a Firenze Lorenzo il Magnifico mentre il Savonarola tonava dal suo pulpito ${ }^{74}$, l'anno in cui i Mori furono cacciati da Granada, il Pronosticon de eversione Europae (che taluno preferisce datare a qualche anno dopo) era forse già stato scritto. Sempre nel 1492 un marinaio, genovese o catalano, sulla base di calcoli nautici errati, a capo di una flottiglia pressoché ridicola, con la testa piena di pessime letture geografiche e di assurdi progetti di crociata-rivincita, scopriva realmente gli apocalittici ecielo nuovo e nuova terrar. Otto anni dopo, nell'aprile del 1500 , si stendeva a Orvieto il contratto che avrebbe permesso a Luca Signorelli di affrescare col suo "Giudizio Universalen la cappella di San Brizio in Duomo. Più tardi, a Roma, sarebbe stata affrescata la Cappella Sistina.

L'Apocalisse segue passo dietro passo il medioevo cristiano, apre l'era moderna, prosegue - magari «laicizzatas in utopia-il suo cammino fino a noi. C'è da stupirsi se, malgrado tutte le slaicizzazionin, ne siamo ancora spaventati, ancora affascinati? $?^{75}$.

74. R. DE MAro, Savonarola, Alessandro VT e il mitto dell'Anticristo, Rivista storica italiana, LXXXII, III, 1970, p. 533-59.

75. Il grande mito dell Anticristo penetra profondamente anche il mondo contemporaneo. Già molte delle opere cit. supra ne danno conto: dal canto nostro ci limitiamo qui a rinviare a V. Soloviev, Il racconto dell'Anticristo, in Idem, I tre dialoghi, Genova, 1975. Il tema dell'Anticristo nella società moderna e xpostmodernas è proposto da M. CENTINI, II ritorno dell'Anticristo, Casale Monferrato, 1996. 